\title{
ANALYTIC DETERmination AND NUMERICAL SimUlation OF TORSIONAL STIFFness OF A RACING CAR FraMe
}

\author{
Michal Skovajsa, Frantisek Sedlacek
}
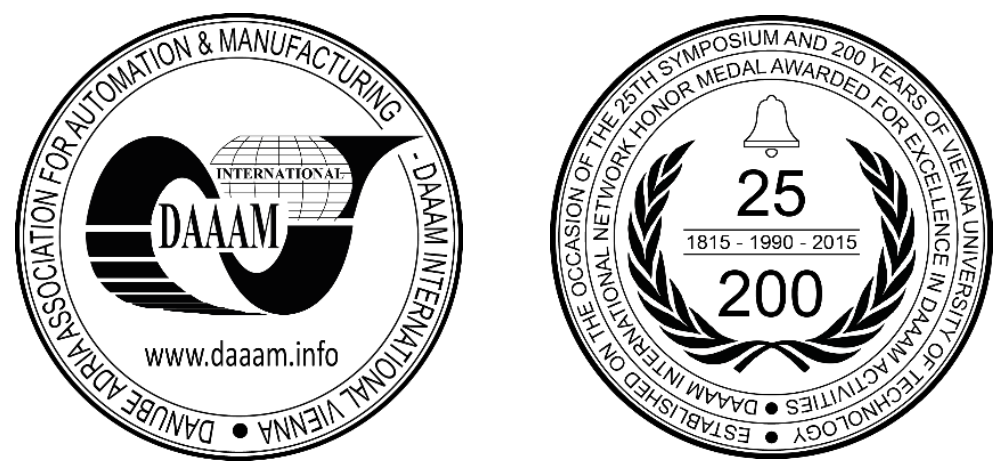

This Publication has to be referred as: Skovajsa, M[ichal] \& Sedlacek, F[rantisek] (2017). Analytic Determination and Numerical Simulation of Torsional Stiffness of a Racing Car Frame, Proceedings of the 28th DAAAM International Symposium, pp.0671-0678, B. Katalinic (Ed.), Published by DAAAM International, ISBN 978-3-902734-11-2, ISSN 1726-9679, Vienna, Austria

DOI: $10.2507 / 28$ th.daaam.proceedings.094

\begin{abstract}
The paper deals with the determination of the required torsional stiffness of the frame of a racing car. The required torsional stiffness of the frame was performed by analytic computation. The analytic computation deals with the transfer of the vertical load of the tyre between the front and rear axle for different roll stiffnesses of axles and torsional stiffnesses of the frame. The analytic computation determines the ratio between the roll stiffness of the axles and the torsional stiffness of frame. NX Nastran 11 software was used for the numerical simulation. The numerical simulation was performed by using four different numerical methods. Each numerical simulation was performed with different boundary conditions.
\end{abstract}

Keywords: Torsional stiffness; frame; numerical simulation; analytic solution

\section{Introduction}

Determining the correct ratio between the roll stiffness of the car and the torsion stiffness of the frame is covered by several publications. [1, 2, 3]. The numerical simulation of the torsion stiffness of the frame is covered by some publications $[5,6]$. These publications do not deal with the comparison of different numerical calculation methods. The real frame of the Formula Student car UWB05 of the University of West Bohemia was used for the simulation. The questions are: What is the required value of the torsional stiffness of the car? Which numerical simulation method should be chosen for computing the torsion stiffness of the frame?

The frame provides the transmission of force between the tyres and the centre of gravity of the car. The frame transfers four type of loads: Torsional load, Lateral load, Vertical load, and Longitudinal load [4]. The torsion stiffness is the most important parameter of a racing car frame. The frame ensures the lateral load transfers between the front and rear axles, thereby influencing the driving stability and even distribution of the vertical loads of individual tyres. This affects the driving stability and equally the distribution of the vertical loads of individual tyres.

Low values of torsional stiffness cause: incorrect distribution of lateral load, incorrect response of the car to chassis changes, big changes in suspension kinematics compared to the designed parameters, increases the dynamic strain on the car, reduces driving stability, increases the fatigue stress of the car. The torsion stiffness of the frame is defined as the ratio of the torsion stiffness of the frame and the roll stiffness of the axles. The torsion stiffness is defined by the static model of the vehicle (Figure 1), where the stiffness of the axles and the frame are replaced by springs [4]. 


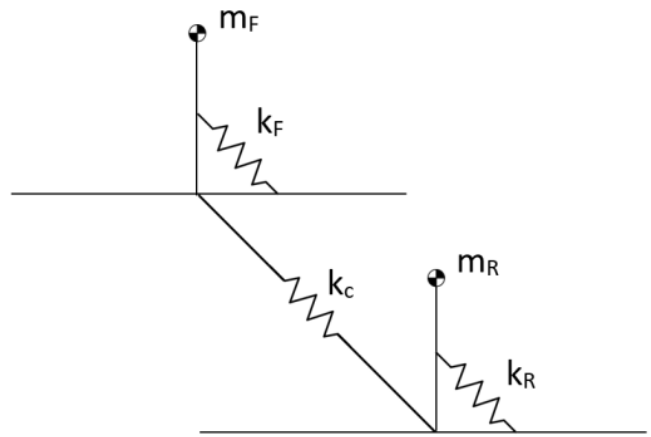

Fig. 1. Static model of the car [2]

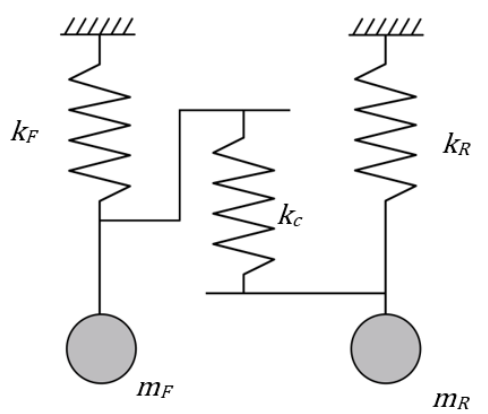

Fig. 2. Static model of the car as a classic spring model

Where $m_{F}$ is load of front axle; $m_{R}$ is load of rear axle; $k_{F}$ is roll stiffness of front axle; $k_{R}$ is roll stiffness of rear axle and $\mathrm{k}_{\mathrm{c}}$ is torsion stiffness of frame. This static vehicle model is replaced by a classic spring system with two points of gravity. (Figure 2). The total torsional stiffness of the car is defined as the sum of the compliance of each element of the vehicle model.

\section{Analytic computation}

The vertical load of the wheels of one axle is identical in the static position of the car. The load on the inner wheel is reduced and the external wheel load is increased if the lateral force (Equation 1) is applied (turning) [1].

$$
\Delta F_{z}=\frac{m h_{C G}}{t} a_{y}
$$

where $\Delta F_{Z}$ is the difference of the vertical load of the car; $m$ is vehicle weight; $h_{C G}$ is centre of gravity height; $t$ is track; $a_{y}$ is acceleration in lateral direction.

Setting assumptions for calculation: Lateral acceleration does not generate roll of the car, Rolling of front and rear axle is independent, The position of the centre of the vehicle and the centre of gravity lies in the longitudinal plane of the car, Unsupported masses are negligible, The front and rear axle track is the same, All norms to the roll axis are vertical.

\subsection{Mathematical model of car with two masses and infinitely stiff frame}

First was defined the mathematical model of a car with two masses and an infinitely stiff frame. This model is described by equations $(2,3,4,5,6,7)[1,4,7]$ and is shown in Figure 3

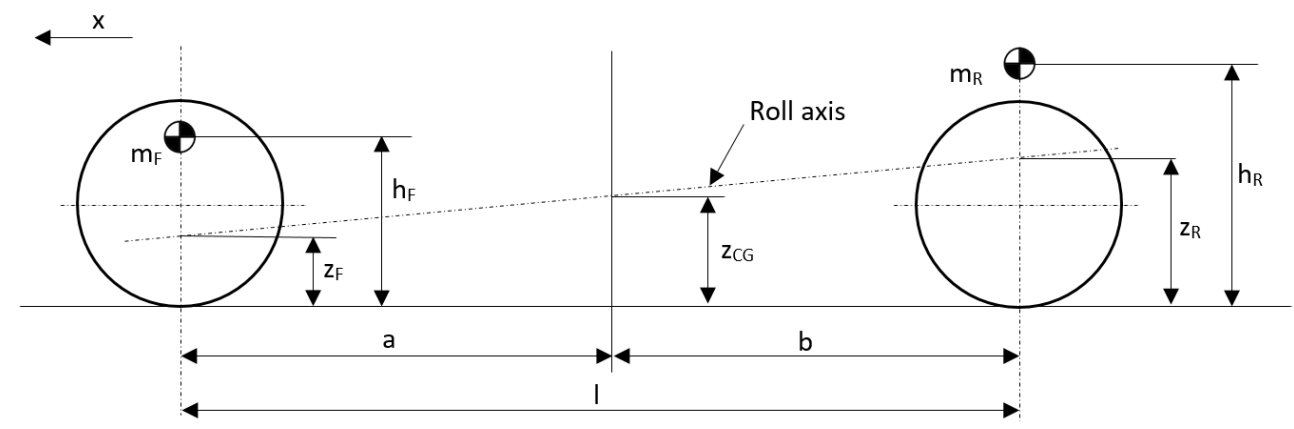

Fig. 3. Two mass model of a car [1]

$$
\begin{aligned}
& m_{F}=\frac{b}{l} m ; m_{R}=\frac{b}{l} m, \\
& d_{F}=h_{F}-z_{F} ; d_{R}=h_{R}-z_{R}, \\
& \Delta F_{z F 0}=\frac{m}{t}\left(\frac{k_{F}}{k_{F}+k_{R}} \frac{b}{l} d_{F}+\frac{k_{F}}{k_{F}+k_{R}} \frac{a}{l} d_{R}+\frac{b}{l} z_{F}\right) a_{y},
\end{aligned}
$$




$$
\Delta F_{z R 0}=\frac{m}{t}\left(\frac{k_{R}}{k_{F}+k_{R}} \frac{b}{l} d_{F}+\frac{k_{R}}{k_{F}+k_{R}} \frac{a}{l} d_{R}+\frac{a}{l} z_{R}\right) a_{y}
$$

where $\Delta F_{Z F O}$ is the difference of the vertical load of the front axle with an infinitely stiff frame; $\Delta F_{R F O}$ is difference of vertical load of the rear axle with infinitely stiff frame; $m$ is vehicle weight; $m_{F}$ is load of front axle; $m_{R}$ is load of rear axle; $I$ is wheelbase; $t$ is track; a is distance between centre of gravity and front axle axis; $b$ is distance between centre of gravity and axis of rear axle; $k_{F}$ is roll stiffness of front axle; $k_{R}$ is roll stiffness of rear axle; $\mathrm{a}_{\mathrm{y}}$ is acceleration in lateral direction; $z_{F}$ is height of roll axis of front axle; $z_{R}$ is height of roll axis of rear axle; $d_{F}$ is distance between centre of gravity and roll axis of front axle; $d_{R}$ is distance between centre of gravity and roll axis of rear axle; $h_{\mathrm{F}}$ is centre of gravity height of front axle; $h_{R}$ is centre of gravity height of rear axle. This model was used for comparison with the model which is described below.

\subsection{Mathematical model of car with two masses and finite stiffness of frame}

The second step was to define the mathematical model of a car with two masses and finite stiffness of the frame. This model is described by equations $(8,9)[1,4,7]$ and is shown in Figure 3

$$
\begin{aligned}
& \Delta F_{z F}=\frac{m}{t}\left(\frac{k_{F}}{k_{F}+\frac{k_{R} k_{C}}{k_{R}+k_{C}}} \frac{b}{l} d_{F}+\frac{\frac{k_{F} k_{C}}{k_{F}+k_{C}}}{k_{R}+\frac{k_{F} k_{C}}{k_{F}+k_{C}}} \frac{a}{l} d_{R}+\frac{b}{l} z_{F}\right) a_{y} \\
& \Delta F_{z R}=\frac{m}{t}\left(\frac{\frac{k_{R} k_{C}}{k_{R}+k_{C}}}{k_{F}+\frac{k_{R} k_{C}}{k_{R}+k_{C}}} \frac{b}{l} d_{F}+\frac{k_{R}}{k_{R}+\frac{k_{F} k_{C}}{k_{F}+k_{C}}} \frac{a}{l} d_{R}+\frac{b}{l} z_{R}\right) a_{y}
\end{aligned}
$$

Where $\Delta F_{Z F}$ is the difference of the vertical load of the front axle with finite stiffness of frame; $\Delta F_{R F}$ is difference of vertical load of the rear axle with finite stiffness of frame; $m$ is vehicle weight; $\mathrm{m}_{\mathrm{F}}$ is load of front axle; $m_{R}$ is load of rear axle; 1 is wheelbase; $t$ is track; $a$ is distance between centre of gravity and front axle axis; $b$ is distance between centre of gravity and axis of rear axle; $k_{F}$ is roll stiffness of front axle; $k_{R}$ is roll stiffness of rear axle; $k_{c}$ is torsion stiffness of frame; $a_{y}$ is acceleration in lateral direction; $z_{F}$ is height of roll axis of front axle; $z_{R}$ is height of roll axis of rear axle; $d_{F}$ is distance between centre of gravity and roll axis of front axle; $d_{R}$ is distance between centre of gravity and roll axis of rear axle; $h_{F}$ is centre of gravity height of front axle; $h_{R}$ is centre of gravity height of rear axle.

\subsection{Comparison of mathematics with infinitely stiff and finitely stiff frame}

The comparison was defined as the difference of the ratios of the vertical loads. The first ratio $X_{F}$ was defined as the fraction of $\Delta F_{Z F}$ which is the difference of the vertical load of the front axle with a finitely stiff frame and $\Delta F_{Z}$ which is the difference of the vertical load of the car. The second ratio $X_{F O}$ was defined as the fraction of $\Delta F_{Z F O}$ which is the difference of the vertical load of the front axle with an infinitely stiff frame and $\Delta \mathrm{F}_{\mathrm{Z}}$ which is the difference of the vertical load of the car.

$$
\begin{aligned}
& X_{F}=\frac{\Delta F_{z F}}{\Delta F_{z}}, \\
& X_{F 0}=\frac{\Delta F_{z F 0}}{\Delta F_{z}},
\end{aligned}
$$

where $X_{F}$ is the ratio change in the vertical load of the front axle with a finitely stiff frame; $X_{F O}$ is the ratio change in the vertical load of the front axle with an infinitely stiff frame.

Graphs 1, 2, 3, 4 were created based on equations $(10,11)[1,4,7]$. Graphs 1 and 2 show the difference of ratio 10 and 11 depending on the torsion stiffness of the frame for different roll stiffnesses of the front axle. Graphs 3 and 4 show the difference of ratios 10 and 11 depending on the roll stiffness of the front axle for different torsion stiffnesses of the frame. Graphs 1 and 3 were created by assuming equal distribution of the load on each axle $\left(m_{F}=m_{R}\right)$. Graphs 2, 4 were created by assuming different distributions of the load on each axle in ratio $47: 53\left(0.53 m_{F}=0.47 m_{R}\right)$. All graphs were created by assuming the same parameters for both axles $\left(Z_{F}=Z_{R} ; h_{F}=h_{R} ; d_{F}=d_{R}\right)$ 


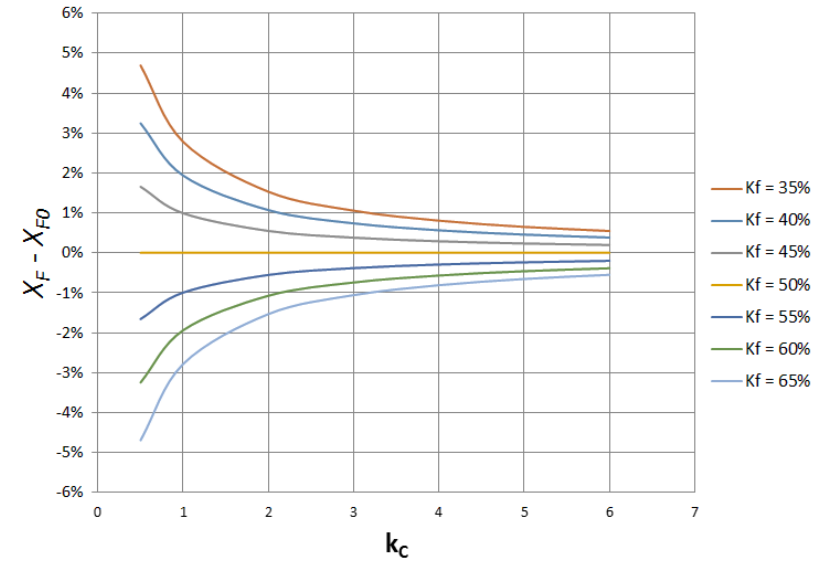

Graph 1. Difference of ratio $X_{F}$ and $X_{F O}$ depending on torsion stiffness of frame for different roll stiffnesses of front axle. For equally distributed load on each axle $\left(m_{F}=m_{R}\right)$.

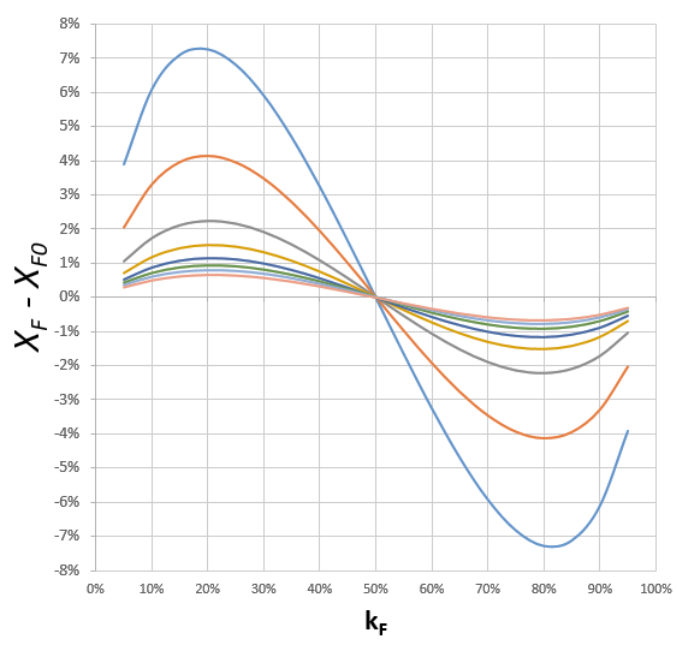

Graph 3. Difference of ratio $X_{F}$ and $X_{F 0}$ depending on roll stiffness of front axle for different torsion stiffnesses of frame. For equally distributed load on each axle $\left(m_{F}=m_{R}\right)$.

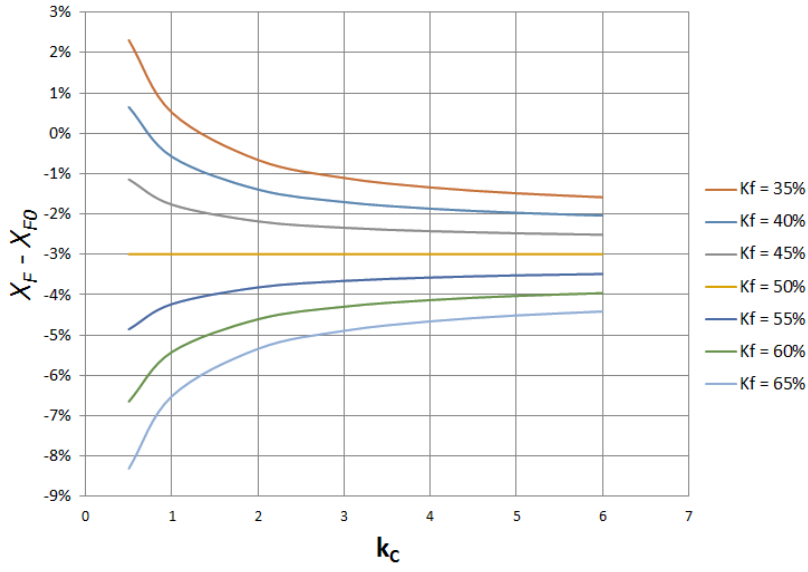

Graph 2. Difference of ratio $X_{F}$ and $X_{F O}$ depending on torsion stiffness of frame for different roll stiffnesses of front axle. For different distribution of load on each axle in ratio $47: 53\left(0,53 m_{F}=0,47 m_{R}\right)$.

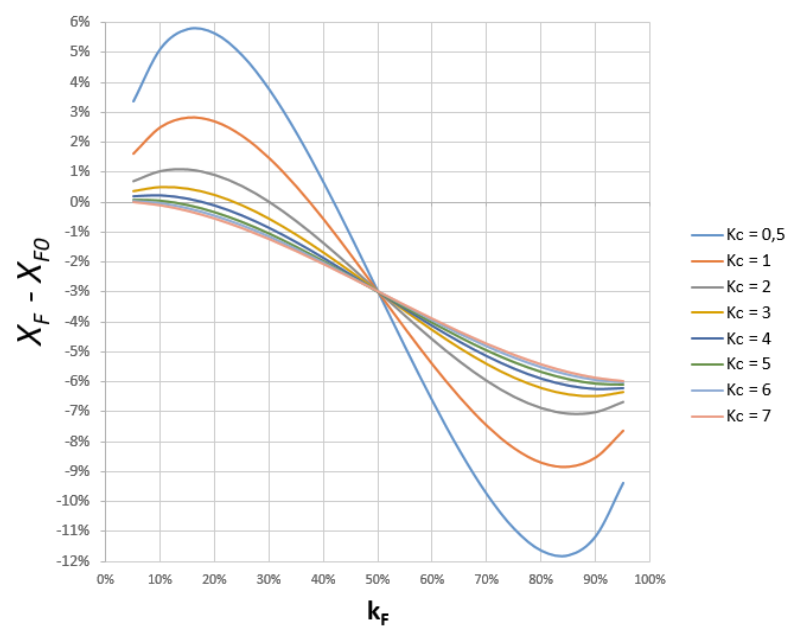

Graph 4. Difference of ratio $X_{F}$ and $X_{F 0}$ depending on roll stiffness of front axle for different torsion stiffnesses of frame. For different distribution of load on each axle in ratio 47:53 $\left(0.53 m_{F}=0.47 m_{R}\right)$.

\begin{tabular}{|c|c|c|c|c|c|c|c|}
\hline & \multicolumn{7}{|c|}{$k_{C}$} \\
\hline & 0,5 & 1 & 2 & 3 & 4 & 5 & 6 \\
\hline$k_{F}=35 \%$ & $4.69 \%$ & $2.78 \%$ & $1.53 \%$ & $1.06 \%$ & $0.81 \%$ & $0.65 \%$ & $0.55 \%$ \\
\hline$k_{F}=40 \%$ & $3.24 \%$ & $1.94 \%$ & $1.07 \%$ & $0.74 \%$ & $0.57 \%$ & $0.46 \%$ & $0.38 \%$ \\
\hline$k_{F}=45 \%$ & $1.66 \%$ & $0.99 \%$ & $0.55 \%$ & $0.38 \%$ & $0.29 \%$ & $0.24 \%$ & $0.20 \%$ \\
\hline$k_{F}=50 \%$ & $0.00 \%$ & $0.00 \%$ & $0.00 \%$ & $0.00 \%$ & $0.00 \%$ & $0.00 \%$ & $0.00 \%$ \\
\hline$k_{F}=55 \%$ & $-1.66 \%$ & $-0.99 \%$ & $-0.55 \%$ & $-0.38 \%$ & $-0.29 \%$ & $-0.24 \%$ & $-0.20 \%$ \\
\hline$k_{F}=60 \%$ & $-3.24 \%$ & $-1.94 \%$ & $-1.07 \%$ & $-0.74 \%$ & $-0.57 \%$ & $-0.46 \%$ & $-0.38 \%$ \\
\hline$k_{F}=65 \%$ & $-4.69 \%$ & $-2.78 \%$ & $-1.53 \%$ & $-1.06 \%$ & $-0.81 \%$ & $-0.65 \%$ & $-0.55 \%$ \\
\hline $\begin{array}{c}\left(X_{F-}-X_{F 0}\right)_{\triangle k F 35 \%}-\left(X_{F}-X_{F 0}\right)_{\triangle k F 65 \%} \\
\left.-\left(\left(X_{F-}-X_{F 0}\right)_{\Delta k F 35 \%}-\left(X_{F-}-X_{F 0}\right)_{\Delta k F 65 \%}\right)_{i k F 35 \%}-\left(X_{F-1}-X_{F 0}\right)_{\Delta k F 65 \%}\right)_{i}\end{array}$ & $9.38 \%$ & $5.56 \%$ & $3.06 \%$ & $2.11 \%$ & $1.61 \%$ & $1.31 \%$ & $1.10 \%$ \\
\hline
\end{tabular}

Table 1. Difference of ratio $X_{F}$ and $X_{F O}$ depending on torsion stiffness of frame for different roll stiffnesses of front axle. For equal distribution of load on each axle $\left(m_{F}=m_{R}\right)$

From all the graphs it was determined that a change of the torsional stiffness of the frame has no affect if the roll stiffness of the axles is the same $\left(k_{F}=k_{R}\right)$. From all the graphs it was determined that a change in the ratio $m_{F} / m_{R}$ does have an effect. The lateral load transfer is increased and the inflection point of the graphs is moved. 
Because the car is loaded with dynamic action and the characteristics of the shock absorbers and progressive springs are nonlinear, then the roll stiffness characteristics of the individual axles are nonlinear too. The amplitude of roll stiffness of the axle was determined to be from $35 \%$ to $65 \%$. Table 1 was created for this range. Table 1 contains the values of the differences and the ratios of the changes of differences of transfer of the lateral forces which is defined as $X_{F}-X_{F 0}$.

A significant reduction of lateral force transfer was achieved with the value of $k_{c}$ in a range from 0.5 to 4 . An improvement of only about $0.5 \%$ was achieved for $k_{c}$ over 4 . For this reason, the final value of torsional stiffness of the frame was determined. The torsional stiffness of the frame is suitably set to a value $4-6$ times the roll stiffness of the axles.

\section{Numerical simulation of torsional stiffness of frame}

The numerical solution was performed on the frame of the Formula Student racing car UWB05. Four solution variants of the numerical simulations of the torsional stiffness of the frame were carried out. The force and constrain for solutions one and two were applied to the frame. For example: The roll stiffness of the UWB05 car is 452 [Nm/deg]. Therefore, the required torsional stiffness of the frame is from $1808-2702[\mathrm{Nm} / \mathrm{deg}]$.

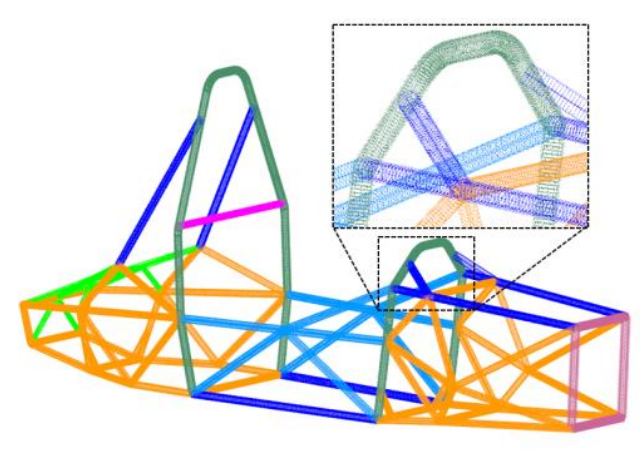

Fig. 4. 1D FE model of frame

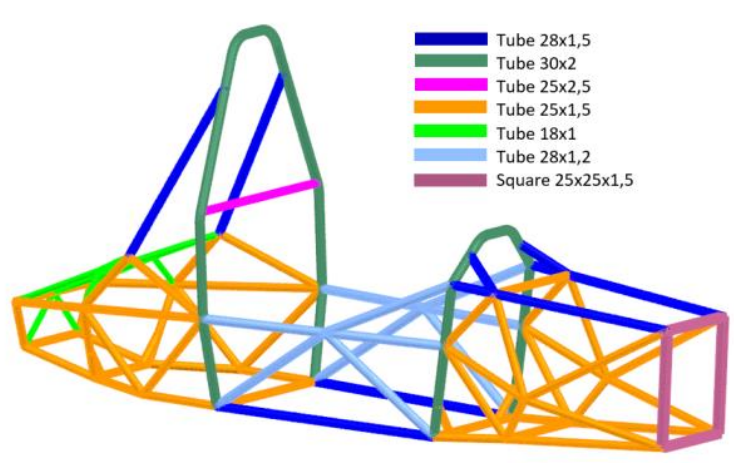

Fig. 5. Physical model of frame

Numerical simulations of the torsional stiffness of the frame were done using the linear structural solver NX Nastran 101 and pre-post-processor Siemens Simcenter $11[8,9]$. A 1D model of the frame was created using 1D fine linear element mesh (cbeam elements) [10]. $5 \mathrm{~mm}$ FE mesh was used for the numeric simulation. The 1D FE model with mesh is shown in Figure 4. The boundary conditions of solution numbers 1 and 2 were applied directly to the frame. The numerical models for solutions 3 and 4 were extended using the model of the suspension, as shown in Figure 6. The connections between each link of the suspension and the suspension with the frame were formed by manual coupling. The manual coupling was set to transfer only the translation to ensure a rotary link. The forces and constrains for solutions 3 and 4 were applied to the suspension. In solution three the suspension was replaced by rigid links. In solution 4 the suspension was replaced by beams links, because in a real car the suspension is flexible. Therefore, they must be included in the simulation of the torsional stiffness of the frame.
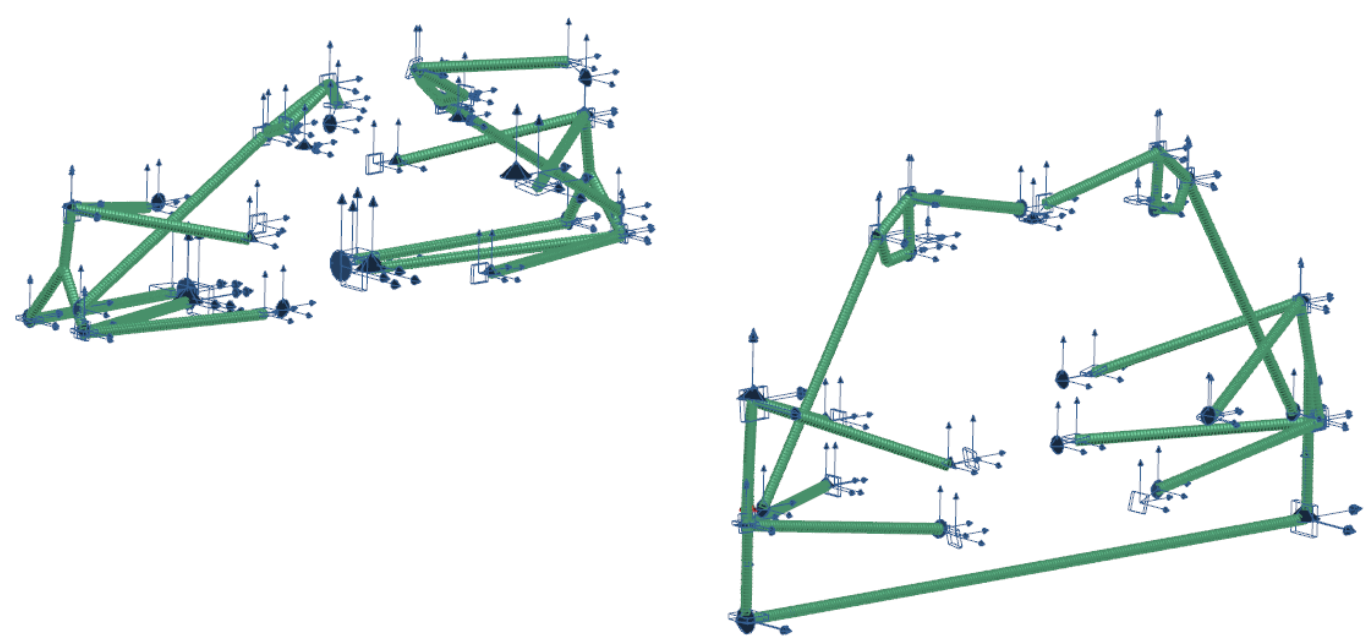

Fig. 6. Numerical model of suspension with manual coupling. 
Solution 1

Setting:

Constrain:

Position: 1, 2, 3, 5 - Fixed Translation ( $x, y, z)$

Forces:

Positon: $6-1000 \mathrm{~N}(z)$

Measurement point:

Position: $7(z)$

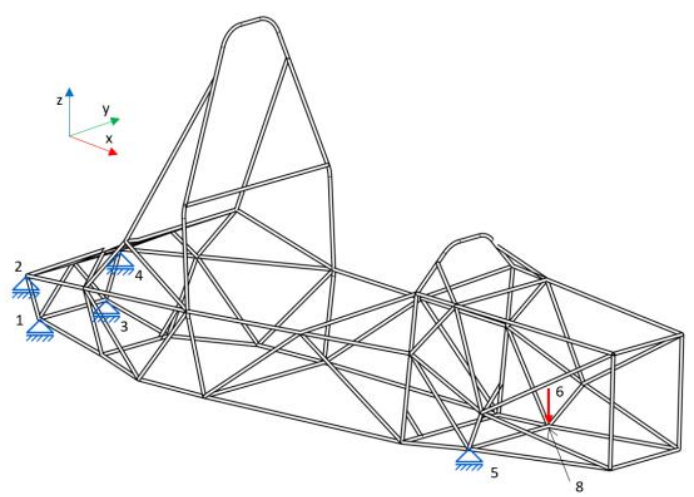

Fig. 7. Constrain and force for solution 1 .

Solution 2

Setting:

Constrain:

Position: 1, 2, 3, 5 - Fixed Translation $(x, y, z)$

Forces:

Positon: $6 \quad-500 N(z)$

Positon: $7+500 \mathrm{~N}(z)$

Measurement point:

Position: $8(z)$

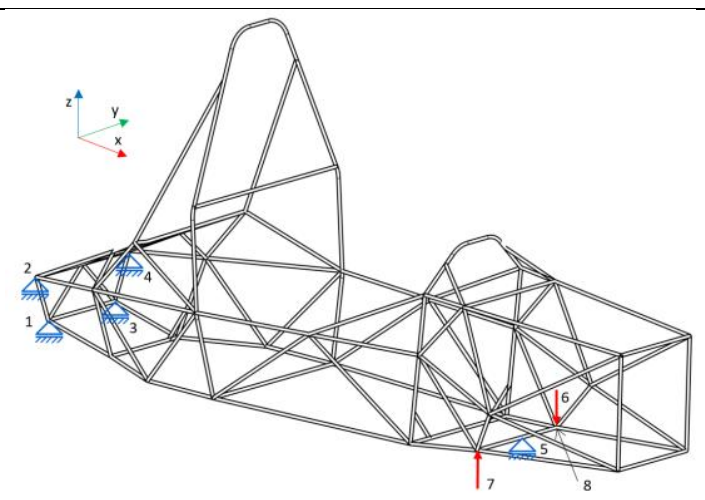

Fig. 8. Constrain and force for solution 2.

Solution 3

Setting:

Constrain:

Position: 1 - Fixed Translation $(x, y, z)$

2 - Fixed Translation $(x, z)$

3 - Fixed Translation $(y, z)$

Forces:

Positon: 4 -1000N (z)

Measurement point:

Position: $5(z)$

Suspension is rigid links

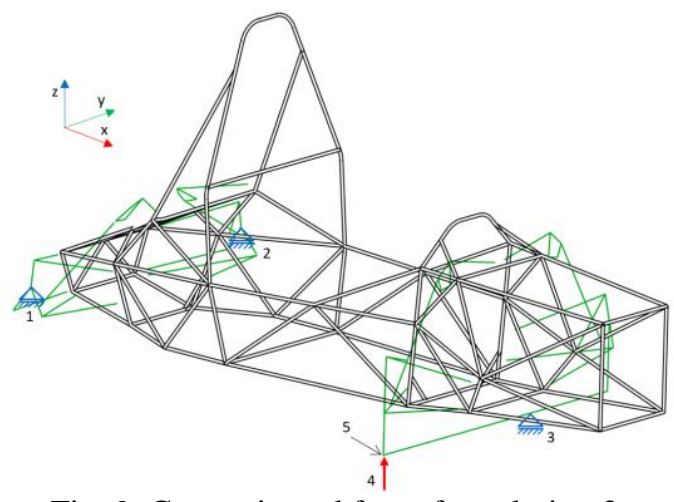

Fig. 9. Constrain and force for solution 3 .

\section{Solution 4}

Setting:

Constrain:

Position: 1 - Fixed Translation $(x, y, z)$

2 - Fixed Translation $(x, z)$

3 - Fixed Translation $(y, z)$

Forces:

Positon: 4 -1000N (z)

Measurement point:

Position: $5(z)$

Suspension is flexible links

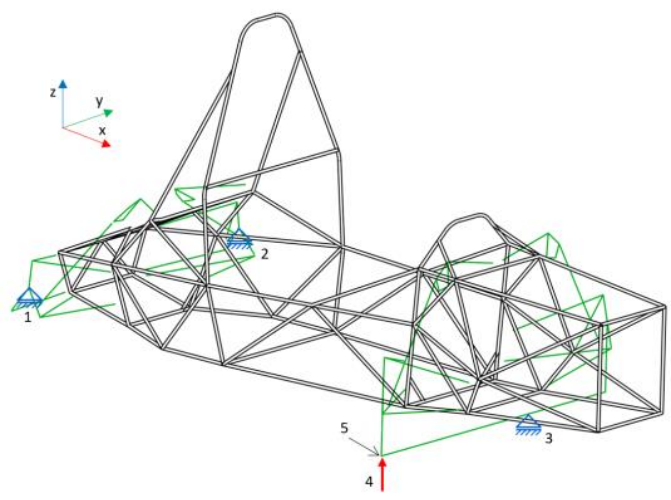

Fig. 10. Constrain and force for solution 4.

The results of the magnitude displacement of structural analysis in direction ' $z$ ' are shown for individual solutions in Fig. 10-13. 


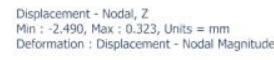
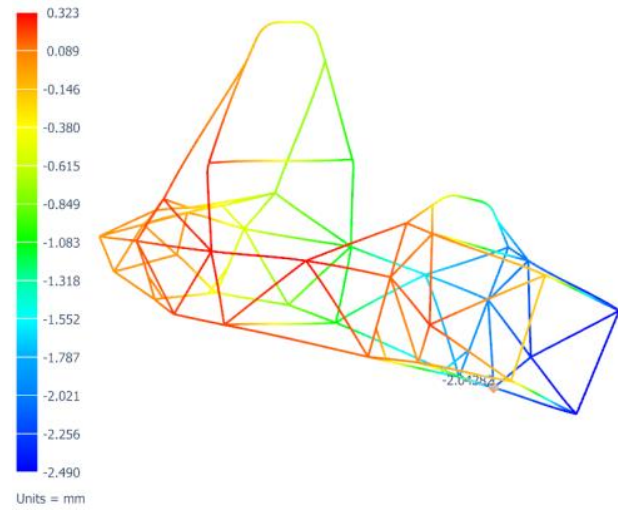

Fig. 11. Result of solution 1.
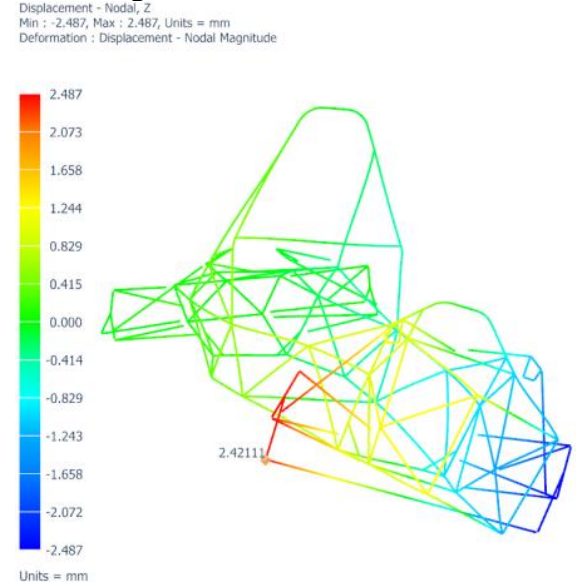

Fig. 13. Result of solution 3 .
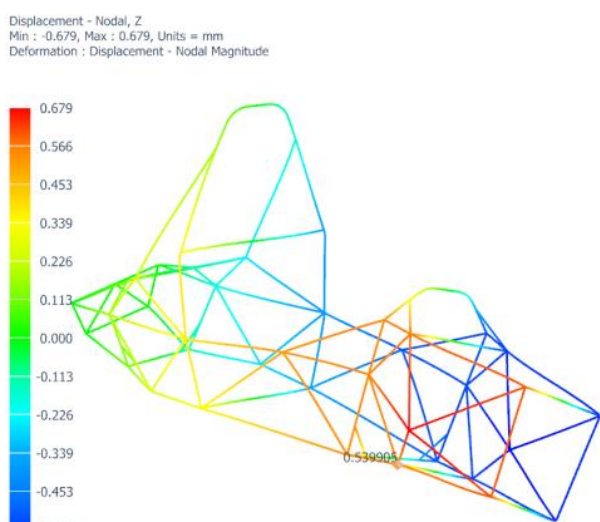

Fig. 12. Result of solution 2.
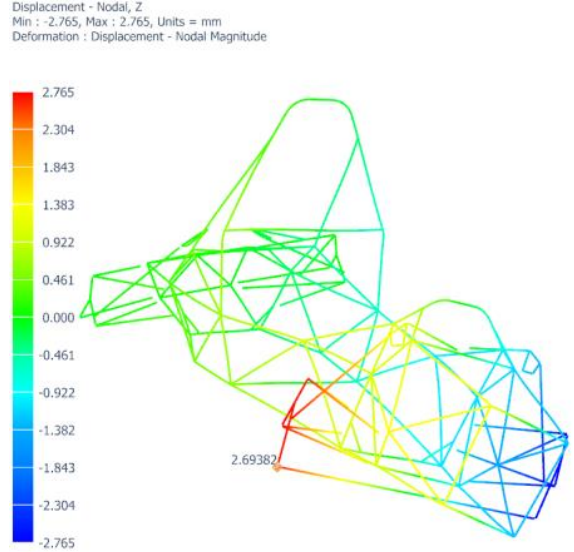

Fig. 14. Result of solution 4.

\section{Comparison of the numerical simulation}

The comparison of the numerical simulations is shown in Table 2. There are very significant differences between the numerical simulations. The maximum difference in torsional stiffness was determined to be 1112.4 [Nm/deg]. Solutions number 1 and 2 are unrealistic in comparison to real car conditions. In solution number 3 the torsional stiffness of the frame was increased by the rigid links of the suspension. In this case the best result is number 4 , where the suspension is connected by flexible links and the constrain and force are most consistent with reality.

\begin{tabular}{|c|c|c|c|c|c|c|c|}
\hline \multirow{2}{*}{ Solution } & \multicolumn{3}{|c|}{ Applied force } & \multicolumn{3}{c|}{ Deformation } & \multirow{2}{*}{ Torsional stiffness } \\
\cline { 2 - 7 } & Force & Arm of force & Torque & Deformation & $\begin{array}{c}\text { Arm of } \\
\text { deformation }\end{array}$ & $\begin{array}{c}\text { Angle of } \\
\text { deformation }\end{array}$ & \\
\hline 1 & $1000[\mathrm{~N}]$ & $460[\mathrm{~mm}]$ & $460[\mathrm{Nm}]$ & $2.04282[\mathrm{~mm}]$ & $460[\mathrm{~mm}]$ & $0.25444[\mathrm{deg}]$ & $1807.8[\mathrm{Nm} / \mathrm{deg}]$ \\
\hline 2 & $2 \times 500[\mathrm{~N}]$ & $180[\mathrm{~mm}]$ & $180[\mathrm{Nm}]$ & $0.5399[\mathrm{~mm}]$ & $180[\mathrm{~mm}]$ & $0.17185[\mathrm{deg}]$ & $1047.4[\mathrm{Nm} / \mathrm{deg}]$ \\
\hline 3 & $1000[\mathrm{~N}]$ & $547.37[\mathrm{~mm}]$ & $547.37[\mathrm{Nm}]$ & $2.69382[\mathrm{~mm}]$ & $547.37[\mathrm{~mm}]$ & $0.25342[\mathrm{deg}]$ & $2159.8[\mathrm{Nm} / \mathrm{deg}]$ \\
\hline 4 & $1000[\mathrm{~N}]$ & $547.37[\mathrm{~mm}]$ & $547.37[\mathrm{Nm}]$ & $2.42111[\mathrm{~mm}]$ & $547.37[\mathrm{~mm}]$ & $0.28197[\mathrm{deg}]$ & $1941.2[\mathrm{Nm} / \mathrm{deg}]$ \\
\hline
\end{tabular}

Table 2. The comparison of the numerical simulations

\section{Conclusion}

The paper deals with the determination of the required torsional stiffness of the frame of a racing car. The questions were: What is the torsional stiffness of the frame required for a specific roll stiffness of the car? Which type of boundary condition is it suitable to use for the numerical simulation of torsional stiffness in solver Nastran 101 in NX 11.

The torsion stiffness of the frame was defined as the ratio of the torsion stiffness of the frame and the roll stiffness of the axles. The torsion stiffness of the frame ensures transfer of the lateral forces between the front and rear axles. The difference of the vertical load of the front axle was defined for frames with infinite and finite stiffness (equations $6,7,8$, 9). The difference of these vertical loads change for both types of frame, as shown in the Graphs $(1,2,3,4)$ and Table 1. From all the graphs it was determined that changing the torsional stiffness of the frame has no effect if the roll stiffnesses 
of the axles are the same $\left(k_{F}=k_{R}\right)$. From all the graphs it was determined that changing the ratio of $\mathrm{m}_{\mathrm{F}} / \mathrm{m}_{\mathrm{R}}$ does have an effect: the lateral load transfer is increased and the inflection point of the graphs is moved. When the racing car is loaded with dynamic action and the characteristics of the shock absorbers and progressive springs are nonlinear, then the roll stiffness characteristics of the individual axles are nonlinear too. The amplitude of the roll stiffness of the axle was determined to be from $35 \%$ to $65 \%$. In this case, a significant reduction of lateral force transfer (about $3.82-0.95 \%$ ) was achieved with a value of $k_{c}$ in a range from 0.5 to 4 . For $k_{c}$ over 4 an improvement of only about $0.5 \%$ was achieved. For this reason, the final value of the torsional stiffness of the frame was determined. The torsional stiffness of the frame is suitably set to a multiple 4 - 6 times the roll stiffness of the axles. For example, if a vehicle with total weight $300 \mathrm{~kg}$ is considered, then the lateral load transfers between the front and rear axle: for $k_{c}=1$ it will be $8.34 \mathrm{~kg}$ and the total distribution will change about $16.68 \mathrm{~kg}$; for $k_{c}=5$ it will be $1.965 \mathrm{~kg}$ and the total distribution will change only about $3.93 \mathrm{~kg}$.

The numerical simulations were performed in four solution variants with different boundary conditions which are described in section 3 . The differences between the numerical simulations are very significant. The maximum difference of torsional stiffness was determined to be 1112.4 [Nm/deg]. Solutions 1 and 2 are unrealistic in comparison to real car conditions. In solution 3 the torsional stiffness of the frame was increased by the rigid links of the suspension. In this case the best result is number 4, where the suspension is made of flexible links and the constrain and force are most consistent with reality. The numerical simulation will have to be verified by an experimental measurement of the real frame. We are currently working on experimental testing of the torsional stiffness of the frame of the UWB05 car, and verification of the numerical simulations to best-fit the numerical simulation to realistic values.

\section{Acknowledgments}

This paper is based on work sponsored by project SGS2016-012 (The complex support of designing technical equipment III.).

\section{References}

[1] Sampo E.; Sorniotti, A.; Crocombe, A. (2010). Chassis Torsional Stiffness: Analysis of tht Influence on Vehicle Dynamic, SAE International, ISSN 0148-7191, USA

[2] Deakin A.; Crolla, D.; Pablo J.; Hanley R. (2000). The Effect Chassis Stiffness of Race Car Handling Balnce, SAE International, 2000-01-3554, USA

[3] Riley W.; George A.; (2002). Design, Analysis and Tesing of a Formula SAE Car Chassis, SAE International, 200001-3300, USA

[4] Milleken F. W.; Millken L. D. (1995). Race Car Vehicle Dynamics, ISBN 1-56091-526-9

[5] Thompson L.; Lampert K.; Law H. (1998). Design of a Twist Fixture to Measure the Torsional Stiffness of a Winston Cup Chassis, SAE International, 983054, USA

[6] Thompson L.; Soni P.; Raju P.; Law H. (1998). The Effects of Chassis Flexibility on Roll Stiffness of a Winston Cup Race Car, SAE International, 983051, USA

[7] Giancarlo G.; Lorenzo M. (2009). The Automotive Chassis: Vol. 2: System Design, Springer, ISBN: 978-1-40208673-1

[8] Siemens PLM, 'NX Nastran 11 User's Guide’. Siemens AG., 2016.

[9] P. Goncharov, Engineering analysis with NX advanced simulation, vol. 2014. Raleigh: Lulu Press.

[10] O. C. Zienkiewicz, R. L. Taylor, and J. Z. Zhu, The Finite Element Method: Its Basis and Fundamentals, Seventh Edition, 7 edition. Amsterdam: Butterworth-Heinemann, 2013. 